\title{
Author Correction: Circulating tumor DNA analysis depicts subclonal architecture and genomic evolution of small cell lung cancer
}

\author{
Jingying Nong1, Yuhua Gong 2,3, Yanfang Guan"2,3, Xin Yi ${ }^{2,3}$, Yuting Yi ${ }^{2,3}$, Lianpeng Chang ${ }^{2,3}$, Ling Yang ${ }^{2,3}$,

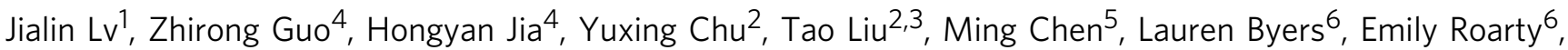 \\ Vincent K. Lam ${ }^{6}$, Vassiliki A. Papadimitrakopoulou ${ }^{6}$, Ignacio Wistuba7, John V. Heymach ${ }^{6}$, Bonnie Glisson ${ }^{6}$, \\ Zhongxing Liao $^{8}$, J. Jack Lee (1) ${ }^{9}$, P. Andrew Futreal ${ }^{10}$, Shucai Zhang ${ }^{1}$, Xuefeng Xia ${ }^{11}$, Jianjun Zhang ${ }^{6,10} \&$ \\ Jinghui Wang ${ }^{1}$
}

Correction to: Nature Communications; https://doi.org/10.1038/s41467-018-05327-w; published online 06 August 2018

The original version of this Article contained an error in Fig. 2, in which the left $y$-axis labels 'tDNA' and 'ctDNA' were inadvertently inverted. This has been corrected in the PDF and HTML versions of the Article.

Published online: 29 January 2019

Open Access This article is licensed under a Creative Commons Attribution 4.0 International License, which permits use, sharing, adaptation, distribution and reproduction in any medium or format, as long as you give appropriate credit to the original author(s) and the source, provide a link to the Creative Commons license, and indicate if changes were made. The images or other third party material in this article are included in the article's Creative Commons license, unless indicated otherwise in a credit line to the material. If material is not included in the article's Creative Commons license and your intended use is not permitted by statutory regulation or exceeds the permitted use, you will need to obtain permission directly from the copyright holder. To view a copy of this license, visit http://creativecommons.org/licenses/by/4.0/.

(c) The Author(s) 2019

\footnotetext{
${ }^{1}$ Department of Medical Oncology, Beijing Chest Hospital, Capital Medical University, Beijing Tuberculosis and Thoracic Tumor Research Institute, 101149 Beijing, China. ${ }^{2}$ Geneplus-Beijing, 102206 Beijing, China. ${ }^{3}$ Geneplus-Beijing Institute, 102206 Beijing, China. ${ }^{4}$ Beijing Key Laboratory for Drug Resistance Tuberculosis Research, Beijing Chest Hospital, Capital Medical University, Beijing Tuberculosis and Thoracic Tumor Research Institute, 101149 Beijing, China. ${ }^{5}$ Department of Radiation Oncology, Zhejiang Cancer Hospital, 310022 Hangzhou, China. ${ }^{6}$ Department of Thoracic/Head and Neck Medical Oncology, University of Texas MD Anderson Cancer Center, Houston, TX 77030, USA. ${ }^{7}$ Department of Translational Molecular Pathology, University of Texas MD Anderson Cancer Center, Houston, TX 77030, USA. ${ }^{8}$ Department of Radiation Oncology, University of Texas MD Anderson Cancer Center, Houston, TX 77030, USA. ${ }^{9}$ Department of Biostatistics, University of Texas MD Anderson Cancer Center, Houston, TX 77030, USA. ${ }^{10}$ Department of Genomic Medicine, University of Texas MD Anderson Cancer Center, Houston, TX 77030, USA. ${ }^{11}$ Houston Methodist Research Institute, Houston, TX 77030, USA. Correspondence and requests for materials should be addressed to S.Z. (email: sczhang6304@163.com) or to J.Z. (email: jzhang20@mdanderson.org) or to J.W. (email: jinghuiwang2006@163.com)
} 